\title{
Etude des relations entre les caractéristiques physico-chimiques des laits de chèvre et leur aptitude à la coagulation par la présure
}

\author{
F. Remeuf ${ }^{1}$, J. Lenoir ${ }^{1}$ et C. Duby ${ }^{2}$ \\ avec la collaboration technique de M.-T. Letilly ${ }^{1}$ et A. Normand 1
}

1 INA Paris-Grignon, laboratoire de recherche de la chaire de technologie, INRA, 78850 ThivervalGrignon;

2 INA Paris-Grignon, laboratoire de biométrie, 16, rue Claude-Bernard, 75231 Paris Cedex 05, France

(reçu le 16 janvier 1989, accepté le 30 juin 1989)

Résumé - Une étude systématique a été entreprise, portant sur la composition protéique et minérale et le comportement vis-à-vis de la présure de 115 échantillons de laits individuels provenant de 40 chèvres.

La grande variabilité des caractéristiques physico-chimiques et technologiques des laits individuels a été mise en évidence. La recherche, par des méthodes statistiques appropriées, des relations entre les caractères physico-chimiques des laits et leur comportement vis-à-vis de la présure, montre qu'un petit nombre de variables physico-chimiques permet d'expliquer une grande partie des variations d'aptitude à la coagulation par la présure des laits. Ces variables sont : les concentrations en caséine totale, en calcium total et colloïdal, le rapport calcium soluble sur calcium total, le diamètre moyen des micelles, et le rapport caséines $\alpha_{s}$ sur caséine $\beta$. D'autre part, il apparaît que l'écart de fermeté entre les coagulums bovin et caprin est essentiellement dû aux différences des caractéristiques micellaires des caséines (diamètre moyen et degré d'hydratation) des deux laits.

L'ensemble des résultats fournit des indications intéressantes pour la recherche de moyens permettant d'améliorer le comportement fromager du lait de chèvre, par correction technologique ou sélection génétique.

lait de chèvre - caractères physico-chimiques - aptitude à la coagulation par la présure

Summary - A study of the relations between physico-chemical characteristics of goat milks and their renneting properties. A systematic study of the protein and mineral composition and rennet coagulating properties of 115 individual milk samples from 40 goats was undertaken.

A great variability in physico-chemical characteristics of individual milks has been observed. Study of the relationships between physico-chemical characteristics of milks and their coagulating properties utilising suitable statistical methods shows that a few physico-chemical variables can explain a major part of the variation in the renneting properties of individual goat milks. These variables are: casein content, total and colloidal calcium content, soluble calcium/total calcium ratio, mean diameter of casein micelles, $\alpha_{s}$ caseins/ $\beta$ casein ratio. On the other hand, the gap between cow's and goat's rennet curd firmness is essentially due to the differences in the physical properties of casein micelles between the two milks (mean diameter and solvation).

These results provide interesting informations on the ways to find out methods for improving the cheesemaking capacity of goat milk, by technological adjustment or genetic selection. 


\section{INTRODUCTION}

Les compositions chimiques globales des laits bovins et caprins sont relativement voisines (Parkash \& Jenness, 1968; Jenness, 1980). En revanche, des différences notables apparaissent lorsqu'on s'intéresse aux caractéristiques physicochimiques fines des deux laits. Ainsi, les proportions relatives des principaux composants de la caséine sont très différentes, le lait de chèvre présentant un rapport caséines $\alpha_{s}$ /caséine $\beta$ inférieur à celui du lait de vache (Richardson et al., 1974; Boulanger, 1976). D'autre part, il existe dans le lait de chèvre une forte variabilité individuelle de la teneur en caséine $\alpha_{s 1}$, liée à l'existence d'un polymorphisme génétique (Grosclaude et al., 1987); certains animaux, qui possèdent le variant dit "nul», produisent un lait totalement dépourvu de caséine $\alpha_{\mathrm{s} 1}$ alors que ceux qui possèdent les variants "forts" (A, B, C) produisent un lait en contenant environ 20$25 \%$ de la caséine entière.

Les caractéristiques micellaires des caséines des deux laits sont également différentes. Le lait de chèvre contient des micelles dont la minéralisation phosphocalcique est plus forte que celle des micelles bovines (O'Connor \& Fox, 1977). A l'opposé, le degré d'hydratation des micelles caprines est plus faible (Thompson et al., 1969). En ce qui concerne les dimensions micellaires, les données disponibles sont très peu nombreuses et plutôt contradictoires. Richardson et al. (1974) ont signalé que le lait caprin contient à la fois plus de grosses et de petites micelles que le lait bovin, ce qui traduit une dispersion plus grande.
Le comportement du lait de chèvre visà-vis de la présure est aussi sensiblement différent de celui du lait de vache. La fermeté du gel-présure est plus faible pour le lait de chèvre (Kalatzopoulos, 1970; AbouDawood \& El-Sawaf, 1977) et Storry et al. (1983) ont montré que, même à teneurs en caséines égales, le coagulum caprin est moins ferme que le coagulum bovin. En revanche il apparaît que le lait de chèvre coagule plus rapidement et atteint la fermeté maximale en un temps plus court que le lait de vache (Steinsholt, 1973; Blattner \& Gallmann, 1980). Les données relatives à l'égouttage tendent à montrer que le gel caprin s'égoutte plus rapidement que le gel bovin (Storry et al., 1983; Nilsen \& Abra hamsen, 1985).

En fromagerie, la faible cohésion du gel caprin peut être à l'origine de pertes de matière sèche sous forme de "fines" dans le lactosérum et il en résulte un rendement moindre que dans le cas du lait de vache.

Les causes de ce comportement différent des laits de chèvre doivent être recherchées dans les différences de caractéristiques physico-chimiques observées entre les deux laits et il serait particulièrement intéressant d'identifier les paramètres ayant une incidence prépondérante sur l'aptitude du lait de chèvre à la coagulation par la présure. Cela permettrait, en effet, d'envisager la mise en œuvre de moyens de correction technologique destinés à améliorer et à régulariser les rendements.

Notre travail avait pour objectif d'apporter des éléments de réponse à ces problèmes et dans ce but, nous nous sommes attachés à l'étude de la composition protéique et minérale de laits individuels et à la recherche des relations entre cette composition et l'aptitude à la coagulation par la présure. 


\section{MATÉRIEL ET MÉTHODES}

\section{Échantillonnage et plan d'expérience}

Les échantillons de lait proviennent du troupeau caprin de la station de nutrition et alimentation INRA de l'INA Paris-Grignon. Un échantillon est constitué par le volume de lait recueilli lors de la traite totale (soir et matin) d'une chèvre individuelle. De l'azothydrate de sodium, à la dose de $40 \mathrm{mg} / \mathrm{l}$, est ajouté au lait du soir afin d'en assurer la conservation à $20^{\circ} \mathrm{C}$ pendant la nuit. Dans le but d'éliminer les laits de mammite, un contrôle est effectué par la méthode CMT, à l'aide du "leucotest" Rhône-Mérieux.

Au total, 115 échantillons, prélevés par randomisation à partir de 40 chèvres des races Alpine et Saanen, ont été analysés sur une période de 2 ans. Les laits ont été prélevés entre le 25 avril et le 25 septembre 1984 puis entre le
12 mars et le 24 septembre 1985. Les animaux ont reçu, pendant l'expérimentation, un régime alimentaire à base de foin de luzerne $(18 \%$ de la matière sèche), de concentré $(37 \%)$ et de pulpes de betteraves ensilées (45\%).

Le mélange des deux traites effectué, la dose d'azothydrate de sodium est ajustée à 40 $\mathrm{mg} / \mathrm{l}$ pour inhiber tout développement microbien et les échantillons sont écrémés par centrifugation à $1500 \mathrm{~g}$ pendant $20 \mathrm{~min}$ à $20^{\circ} \mathrm{C}$.

Toutes les déterminations sont ainsi réalisées sur des laits n'ayant subi aucune modification de leurs caractéristiques originelles.

\section{Analyses chimiques}

Toutes les analyses chimiques sont réalisées en double sur chaque échantillon. Le Tableau I donne la liste des variables mesurées et leurs abréviations.

Tableau I. Liste des variables et abréviations.
Abréviations
Explications

ASB

AST

BET

CAC

CAI

CAT

CNS

CNT

CPI

CST

DHC

DHY

DMO

FMG

KAP

MIC

MIP

PIC

PSE

PST

TPR

VRG rapport proportion de caséines $\alpha_{\mathrm{s}}$ / proportion de caséine $\beta$

proportion de caséines $\alpha_{\mathrm{s}}$ totales (\%)

proportion de caséine $\beta$ (\%)

concentration en calcium colloïdal ( $\mathrm{mg} / \mathrm{l})$

concentration en calcium ionisé (mg/)

concentration en calcium total ( $\mathrm{mg} / \mathrm{l})$

concentration en caséines solubles ( $\mathrm{g} / \mathrm{l})$

concentration en caséines totales $(\mathrm{g} / \mathrm{l})$

rapport calcium / phosphore inorganique colloïdaux

rapport calcium soluble / calcium total (\%)

degré d'humidité du caillé centrifugé ( $\mathrm{HH}_{2} \mathrm{O} / \mathrm{gMS}$ )

degré d'hydratation des micelles ( $\mathrm{gH}_{2} \mathrm{O} / \mathrm{gMS}$ )

diamètre moyen des micelles $(\mathrm{nm})$

fermeté du gel présure (unités arbitraires, u.a.)

proportion de caséine $\mathrm{K}(\%)$

rapport calcium colloödal / concentration en caséines $(\mathrm{mg} / \mathrm{g})$

rapport phosphore inorganique colloïdal / concentration en caséines ( $\mathrm{mg} / \mathrm{g})$

concentration en phosphore inorganique colloïdal $(\mathrm{mg} / \mathrm{l})$

poids de sérum égoutté $(\mathrm{g} / \mathrm{ml}$ de lait)

rapport phosphore inorganique soluble / phosphore inorganique total (\%)

temps de prise ( $\mathrm{min})$

vitesse de raffermissement du gel (u.a./min) 


\section{Fractionnement et dosage des matières azotées}

Les dosages d'azote comportent la minéralisation de l'échantillon par un mélange sulfoperchlorique, puis le dosage de l'ammoniaque par colorimétrie, selon la réaction de Berthelot, à l'aide d'un analyseur automatique Technicon.

L'azote total (AT) est dosé directement sur le lait, l'azote non protéique (ANP) sur le filtrat séparé après défécation du lait par l'acide trichloracétique (TCA) à 12\%. L'azote soluble (AS) est dosé sur le sérum résultant de la précipitation du lait à pH 4,6 et l'azote non centrifugeable (ANC) sur le surnageant d'ultracentrifugation $\left(80000 \mathrm{~g}, 1 \mathrm{~h}, 20^{\circ} \mathrm{C}\right)$. A partir de ces déterminations, sont calculées :

- la concentration en protéines : PRO $=$ (AT. ANP) $\times 6,38$;

- la concentration en caséines : CNT $=$ (ATAS) $\times 6,38$;

- la concentration en caséines non centrifugeables : CNS $=($ ANC-AS) $\times 6,38$.

\section{Détermination des proportions relatives des différentes protéines par électro- phorèse}

Les caséines ainsi que la $\beta$-lactoglobuline et l' $\alpha$ lactalbumine sont séparées par électrophorèse sur gel de polyacrylamide-agarose à $\mathrm{pH} 8,6$, selon la méthode de Uriel (1966), à partir de caséine présure et de sérum présure. Pour la préparation des solutions mères, $20 \mathrm{ml}$ de lait, placés dans un tube de centrifugeuse, sont additionnés de $200 \mu \mathrm{l}$ de présure Boll à $520 \mathrm{mg}$ de chymosine diluée au 1/10. Après coagulation de $1 \mathrm{~h}$ à $30^{\circ} \mathrm{C}$ l'échantillon est centrifugé à $9000 \mathrm{~g}$ pendant $10 \mathrm{~min}$ à $20^{\circ} \mathrm{C}$. Le surnageant constitue la solution mère des protéines du sérum. Le culot est lavé à 3 reprises à l'eau distillée, puis dissous dans $10 \mathrm{ml}$ d'urée 9M. La solution obtenue représente la solution mère de caséines.

La réalisation de l'électrophorèse à partir de caséine présure se justifie par le fait que, contrairement à la caséine $K$, la paracaséine $K$ migre en une seule bande, à un endroit différent des caséines $\alpha_{S}$ (Assenat, 1967). Dans le calcul des pourcentages relatifs des caséines, le fac- teur de correction égal au rapport des poids moléculaires des chaînes peptidiques de la caséine $K$ et de la paracaséine $K(1,58)$, est appliqué afin d'obtenir l'estimation de la proportion de caséine $\mathrm{K}$.

La migration, d'une durée totale de $180 \mathrm{~min}$, est suivie d'une précipitation des protéines dans un tampon TCA $11,5 \%$ acide sulío-salicylique $3,5 \%(p / v)$, puis d'une coloration des gels dans une solution de bleu de Coomassie à $0,25 \%$. Les proportions relatives des différents composants séparés sont estimées à l'aide d'un densitomère à laser LKB Ultroscan relié à un enregistreur Spectra-physics SP 4100.

\section{Dosage des différentes formes de cal- cium et de phospore inorganique}

Les phases soluble et colloïdale sont séparées par ultracentrifugation à $80000 \mathrm{~g}$ pendant $1 \mathrm{~h}$ à $20^{\circ} \mathrm{C}$

\section{Dosages du calcium}

Le calcium total et le calcium soluble sont dosés à l'aide du calcimètre Corning 940 . Les prises d'échantillon sont respectivement de $20 \mu \mathrm{l}$ de lait et de $100 \mu \mathrm{l}$ de surnageant d'ultracentrifugation. Le calcium colloïdal est calculé par différence.

Le calcium ionisé est dosé directement sur le lait à l'aide d'une électrode ionique spécifique Metrohm E $301 \mathrm{Ca}$, reliée à un $\mathrm{pH}$-mètre $\mathrm{Me}$ trohm 654. La mesure est effectuée sur $50 \mathrm{ml}$ d'échantillon maintenus à $25^{\circ} \mathrm{C}$ et additionnés de $1 \mathrm{ml}$ d'une solution de $\mathrm{KCl} 4 \mathrm{M}$ destinée à standardiser la force ionique. Le potentiel mesuré est relié à la concentration en ions calcium par l'intermédiaire d'une courbe-étalon établie à l'aide de solutions de $\mathrm{Ca} / \mathrm{Cl}_{2}$ de concentrations connues.

\section{Dosages du phosphore inorganique}

Le phosphore inorganique total (PIT) et le phosphore inorganique soluble (PIS) sont déterminés sur les filtrats thichloracétiques à $12 \%$ obtenus à partir du lait (PIT) et du surnageant 
d'ultracentrifugation (PIS), par dosage colorimétrique au réactif nitrovanadomolybdique, à l'aide d'un analyseur Technicon.

\section{Détermination des caractéristiques mi- cellaires}

Les dimensions des micelles sont déterminées à l'aide d'un analyseur de particules Coultronics N4 par mesure du coefficient de diffusion et calcul d'après la loi de Stokes-Einstein (Lin et al., 1971). On place dans la cuve de mesure $20 \mu$ l de lait et $3 \mathrm{ml}$ d'ultrafiltrat du même lait obtenus à l'aide d'un module Amicon équipé de membranes YM 10 et sous une pression d'azote de 3,5 bars. Les paramètres de fonctionnement choisis sont les suivants :

- température : $20^{\circ} \mathrm{C}$;

— viscosité : $0,0125 \mathrm{P}(=1,25 \mathrm{mPa} . \mathrm{s})$;

- indice de réfraction : 1,33 ;

— temps global de mesure : $75 \mathrm{~s}$;

— temps d'échantillonnage : $50 \mu \mathrm{s}$.

L'appareil donne directement la valeur du diamètre moyen des micelles (DMO).

Le degré d'hydratation des micelles est déterminé selon la méthode de Thompson et al. (1969), par mesure de l'humidité du culot d'ultracentrifugation à $80000 \mathrm{~g}$ pendant $1 \mathrm{~h}$ à $20^{\circ} \mathrm{C}$.

$\mathrm{Au}$ total, 18 variables permettent d'identifier les caractéristiques physicochimiques des laits.

\section{Aptitude des laits à la coagulation par la présure}

\section{Cinétique de coagulation par la présure}

La courbe d'évolution des caractères rhéologiques du lait emprésuré en fonction du temps est établie à l'aide d'un torsiomètre de Plint (Burnett \& Scott Blair, 1963), muni d'un module-plongeur à 4 pales en croix, et ayant fait l'objet d'une automatisation semblable à celle préconisée par Gervais (1979).
La mesure est réalisée sur $800 \mathrm{~g}$ de lait placés dans un bécher de 2 l et emprésurés à la dose de $25 \mathrm{ml}$ pour 100 I (présure Boll à $520 \mathrm{mg}$ de chymosine par I). Durant toute la coagulation, le lait est maintenu à $30^{\circ} \mathrm{C}$ dans un bainmarie thermostaté.

A partir de la courbe de coagulation, sont calculés les paramètres suivants:

- le temps de prise (TPR) = laps de temps s'écoulant entre l'emprésurage et le début du raffermissement;

- la vitesse de raffermissement du gel (VRG) = pente de la tangente à l'origine, calculée par régression linéaire sur les 5 premières min du raffermissement;

- la fermeté maximale (FMG) $=$ ordonnée maximale de la courbe.

\section{Aptitude à l'égouttage}

$20 \mathrm{ml}$ de lait, placés dans un tube de centrifugation de $50 \mathrm{ml}$ et portés à $30^{\circ} \mathrm{C}$, sont emprésurés comme il est indiqué précédemment.

Après un temps de coagulation de $1 \mathrm{~h} 30$ la séparation du sérum et du caillé est réalisée directement, sans découpage préalable, par centrifugation à $6000 \mathrm{~g}$ pendant $10 \mathrm{~min}$. Sont alors mesurés le poids de sérum égoutté (PSE) et le degré d'humidité du caillé centrifugé (DHC).

$\mathrm{Au}$ total, cinq variables permettent de caractériser le comportement des laits vis-à-vis de la présure: TPR, VRG, FMG, PSE, DHC.

\section{Traitement des résultats}

Le traitement statistique des résultats a été effectué à l'aide du logiciel BMDP (Biomedical Computer Programs, Ed 1981, University of (California) implanté sur le système Multics du centre de traitement de l'information scientifique (CTIS) de I'INRA à Jouy-en-Josas. Nous avons utilisé les programmes 1D (description de données), 9R (régression multiple), 6M (analyse de corrélation canonique). 


\section{RÉSULTATS}

\section{Caractéristiques des échantillons analysés}

Les caractéristiques physico-chimiques moyennes, les valeurs extrêmes et les coefficients de variation observés sur les 115 échantillons analysés sont indiqués dans le Tableau II. Le Tableau III présente les données similaires, concernant le comportement des laits vis-à-vis de la présure. Ces résultats confirment les observations faites précédemment sur un nombre d'échantillons plus faible (Remeuf \& (Le-) noir, 1985), et ils sont en accord avec la plupart des résultats publiés dans la littérature (e.g. Parkash \& Jenness, 1968; Jenness, 1980; Ramos \& Juares, 1981; Le Mens, 1983).

On relève notamment une variabilité importante des caractéristiques physicochimiques et technologiques des échantillons. Ainsi, la teneur en caséines varie du simple au double, la proportion d'azote non protéique dans l'azote total se situe dans une échelle de 1 à 4 , la concentration en caséine soluble et la proportion de caséines $\alpha_{\mathrm{s}}$ présentent, respectivement, des coefficients de variation de 83,4 et

Tableau II. Caractères physico-chimiques des échantillons.

\begin{tabular}{|c|c|c|c|c|c|c|}
\hline \multirow{5}{*}{$\begin{array}{l}\text { Concentration en protéines ( } \mathrm{g} / \mathrm{l}) \\
\text { CNT } \\
\text { Azote non protéique (\% N total) } \\
\text { CNS / CNT }(\%)\end{array}$} & \multicolumn{2}{|c|}{$\begin{array}{l}\text { Valeurs } \\
\text { moyennes } \\
\text { (1) }\end{array}$} & \multicolumn{2}{|c|}{$\begin{array}{l}\text { Valeurs } \\
\text { extrêmes }\end{array}$} & \multirow{2}{*}{$\begin{array}{c}\begin{array}{c}\text { Coefficients } \\
\text { de variation } \\
(\%)\end{array} \\
12,0\end{array}$} & \multirow{2}{*}{$\begin{array}{l}\begin{array}{c}\text { Lait de } \\
\text { vache } \\
\text { (2) }\end{array} \\
32,0\end{array}$} \\
\hline & $28,1 \pm$ & 0,61 & 19,1 & $-36,8$ & & \\
\hline & $22,0 \pm$ & 0,50 & 15,8 & $-30,8$ & 12,8 & 27,0 \\
\hline & $6,4 \pm$ & 0,36 & 3,08 & $-13,2$ & 26,0 & 4,5 \\
\hline & $8,0 \pm$ & 1,34 & 1,1 & $-40,7$ & 77,3 & 5,7 \\
\hline Caséines $\alpha_{s}(\%)$ & $27,0 \pm$ & 1,20 & 7 & 42 & 23,9 & 50,0 \\
\hline Caséine $\beta(\%)$ & $55,0 \pm$ & 1,13 & 43 & 73 & 11,1 & 36,0 \\
\hline Caséine K (\%) & $18,0 \pm$ & 0,72 & 9 & -29 & 21,8 & 14,0 \\
\hline$\alpha$-lactalbumine/ $\beta$-lactoglobuline & $0,52 \pm$ & 0,035 & 0,24 & $-1,08$ & 34,1 & 0,40 \\
\hline DHY & $1,75 \pm$ & 0,02 & 1,43 & $-2,05$ & 7,1 & 1,90 \\
\hline DMO & $255 \pm$ & 3,15 & 201 & -290 & 6,8 & $175^{(3)}$ \\
\hline CAT & $1150 \pm$ & 26 & 880 & -1570 & 12,1 & 1200 \\
\hline CAC/CAT (\%) & $68,7 \pm$ & 0,90 & 55,8 & $-77,8$ & 15,3 & 65,0 \\
\hline CAI & $130 \pm$ & 5 & 71 & -219 & 21,0 & 120 \\
\hline Phosphore inorganique total $(\mathrm{g} / \mathrm{l})$ & $0,65 \pm$ & 0,02 & 0,45 & $-1,00$ & 17,6 & 0,65 \\
\hline $\begin{array}{l}\text { Phosphore inorganique colloîdal } \\
\text { (\% P. inorganique total) }\end{array}$ & $51 \pm$ & 1,29 & 32,5 & $-71,8$ & 14,3 & 50 \\
\hline $\begin{array}{l}\text { Ca colloïdal/caséine }(\mathrm{mg} / \mathrm{g}) \\
\mathrm{P} \text {. inorganique colloïdal/caséine }\end{array}$ & $36,0 \pm$ & 1,03 & 18,9 & $-60,8$ & 15,4 & 28,9 \\
\hline $\begin{array}{l}(\mathrm{mg} / \mathrm{g}) \\
\mathrm{pH}\end{array}$ & $\begin{array}{l}15,1 \pm \\
6,71\end{array}$ & 0,60 & $\begin{array}{r}6,1 \\
6,45\end{array}$ & $\begin{array}{l}-27,4 \\
-6,90\end{array}$ & $\begin{array}{r}20,9 \\
6,6\end{array}$ & $\begin{array}{c}12,0 \\
6,6 \text { (3) }\end{array}$ \\
\hline
\end{tabular}

(1) : Moyenne sur 115 échantillons. Les intervalles de confiance sont calculés au risque d'erreur $\alpha=5 \%$.

(2) : Compilation de données de la littérature.

(3) : Moyenne sur 48 échantillons de laits de vache individuelles (race Frisonne-Holstein). 
Tableau III. Comportement des laits vis-à-vis de la présure.

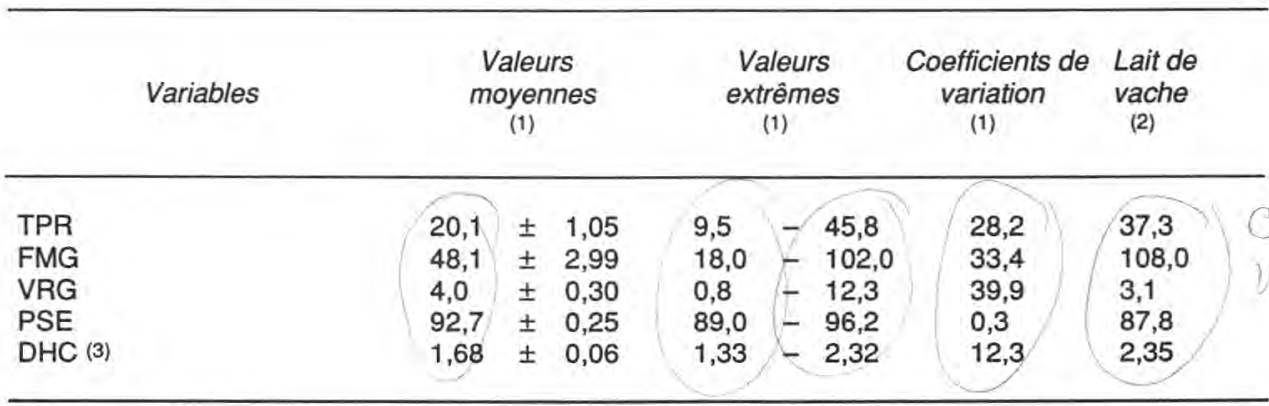

(1) Moyenne sur 115 échantillons. Les intervalles de confiance sont calculés au risque d'erreur $\alpha=5 \%$.

(2) Moyenne des mesures effectuées sur 3 laits de mélange issus d'un troupeau Frison-Holstein.

(3) Valeurs obtenues sur 57 échantillons.

$23,9 \%$. Au niveau des paramètres de la coagulation, le temps de prise et la fermeté de gel se situent dans une échelle d'environ 1 à 5 et la vitesse de raffermissement dans un intervalle de 1 à 15 . Le facteur individu joue un rôle essentiel dans la variabilité observée. Une analyse de variance effectuée à partir d'animaux pour lesquels nous disposions de plusieurs échantillons de lait prélevés à des stades différents de la lactation a montré que, pour la plupart des caractères, la variance résiduelle intraindividuelle est sensiblement inférieure à la variance interindividuelle.

Ces données mettent également en évidence des différences notables entre les laits de chèvre et de vache en ce qui concerne certains caractères. Outre les proportions des caséines, qui sont très différentes notamment pour les caséines $\alpha_{\text {s }}$ et $\beta$, on note un diamètre moyen plus élevé, un degré d'hydratation moindre et une minéralisation plus importante pour les micelles caprines.

Pour ce qui est de l'aptitude à la coagulation par la présure, le lait de chèvre $a$ un comportement qui le distingue nettement du lait de vache. II coagule en un temps plus court, le gel se raffermit plus rapidement, mais il atteint une fermeté maximale nettement inférieure à celle du gel bovin.

\section{Étude des relations entre certaines ca- ractéristiques physico-chimiques}

Le caractère dynamique de la micelle implique que les différentes caractéristiques qui la concernent (dimensions, degré d'hydratation, minéralisation, composition caséinique...) présentent entre elles des inter-relations. Afin de préciser ce point, nous avons cherché à relier par régression multiple les valeurs du diamètre moyen et du degré d'hydratation des micelles aux autres caractères physicochimiques mesurés. Les variables explicatives intervenant dans les équations de régression sont indiquées dans le Tableau IV.

\section{Dimensions des micelles}

Le diamètre moyen des micelles est correlé de manière très significative avec la 
Tableau IV. Régressions multiples entre les caractéristiques micellaires (dimensions et degré d'hydratation des micelles) et les caractères physicochimiques des laits.

\begin{tabular}{lllll}
\hline & \multicolumn{2}{c}{ DMO } & DHY \\
\hline Principales variables & ASB & $\begin{array}{l}(-0,561) \\
(-0,425)\end{array}$ & MIC & $(-0,408)$ \\
explicatives & CNT & MIP & $(-0,247)$ \\
$\begin{array}{l}\text { (coefficients de } \\
\text { régression standard) (1) }\end{array}$ & CAI & $(-0,299)$ & AST & $(-0,168)$ \\
coefficient de corrélation & MIC & $(-0,231)$ & & \\
multiple associé au modèle & & & & \\
\hline
\end{tabular}

(1) Les coefficients de régression standards attribués, à chaque variable dans le modèle, sont les coefficients de régression multipliés par le rapport écart type de la variable explicative sur écart type de la variable expliquée. Ces coefficients peuvent être comparés entre eux du fait de la pondération apportée par l'écart type qui nivelle les différences d'ordre de grandeur entre les valeurs prises par les différentes variables.

concentration en caséines totales : les dimensions micellaires augmentent lorsque la teneur en caséines décroît. Cet effet doit être rapproché des variations de composition car, avec l'accroissement du taux de caséines, il y a accroissement du rapport $\alpha_{s} / \beta$; or les dimensions des micelles sont également liées aux proportions relatives des caséines, les petites micelles présentant un rapport $\alpha_{s} / \beta$ plus élevé que les grosses. En revanche, il n'est pas relevé de relation entre la proportion de caséine $K$ et le diamètre moyen des micelles.

Le diamètre moyen des micelles présente également une corrélation positive avec la concentration en calcium ionisé et une corrélation négative avec le rapport calcium colloïdal/caséine.

\section{Degré d'hydratation des micelles}

Le degré d'hydratation des micelles est correlé négativement avec la minéralisation des micelles, exprimée par la quantité de calcium ou de phosphore inorganique colloïdaux par gramme de caséine. Lorsque la minéralisation augmente, le degré d'hydratation diminue. Nous relevons également une corrélation négative entre le degré de solvatation des micelles et la proportion de caséines $\alpha_{\mathrm{s}}$ totales.

\section{Etude des relations entre les para- mètres de l'aptitude à la coagulation par la présure}

Le Tableau V donne la valeur des coefficients de corrélation simples calculés entre les différentes variables caractérisant le comportement des laits vis-à-vis de la présure. On relève la liaison très étroite existant entre la fermeté du gel et sa vitesse de raffermissement. En d'autres termes, un gel qui se raffermit rapidement a tendance à atteindre une fermeté maximale plus élevée qu'un gel se raffermissant lentement. Le lait correspondant aura aussi tendance à coaguler en un temps 
Tableau V. Coefficients de corrélation entre les différents paramètres de la coagulation $(P<0,05)$.

\begin{tabular}{lrrcc}
\hline & VRG & FMG & PSE & DHC \\
\hline TPR & & & & $*$ \\
VRG & $-0,56$ & $-0,37$ & $-0,44$ & $*$ \\
FMG & & 0,83 & $-0,57$ & $-0,49$ \\
PSE & & & & $*$ \\
\hline
\end{tabular}

* Coefficients de corrélation non significativement différents de 0.

plus court, comme l'indique le coefficient de corrélation négatif hautement significatif relevé entre la vitesse de raffermissement du gel et le temps de prise. Les corrélations observées entre les caractères rhéologiques des gels et leur aptitude à l'égouttage suggèrent qu'un coagulum libère d'autant moins de sérum que le gel est plus ferme. Cette liaison est probablement due au fait que le gel ferme provient en général d'un lait riche en protéines et donc relativement pauvre en eau. II aura donc tendance à libérer une quantité de lactosérum moins importante. D'ailleurs, nos résultats ne permettent pas de mettre en évidence l'existence d'une corrélation significative entre les caractères rhéologiques du gel et le degré d'humidité du caillé centrifugé, critère plus représentatif de l'aptitude réelle du coagulum à la rétention du lactosérum.

\section{Etude des relations entre les caractères physico-chimiques des laits et leur comportement vis-à-vis de la présure}

Cette étude a été menée en utilisant deux méthodes statistiques : la régression multiple et l'analyse de corrélation canonique. La première a permis d'établir des équations reliant chacun des paramètres de l'aptitude à la coagulation par la présure à l'ensemble des caractères physicochimiques. La seconde méthode complète ces résultats par une approche plus synthétique visant à mettre en évidence les variables physicochimiques ayant une influence déterminante sur le comportement global des laits vis-à-vis de la présure.

\section{Régressions multiples}

Le Tableau VI présente les résultats des régressions relatives aux 5 paramètres technologiques. Elles permettent de relever les corrélations significatives qui existent entre ces paramètres et les caractères physico-chimiques des laits.

Temps de prise

Différentes variables caractérisant la fraction minérale ont une influence significative sur le temps de prise. Celui-ci diminue lorsque les concentrations en calcium total et en calcium ionisé augmentent, et lorsque le rapport phosphore inorganique soluble sur phosphore inorganique total croît. Une corrélation positive est mise en évidence entre le temps de prise et le rapport caséines $\alpha_{s}$ /caséine $\beta$. 
Tableau VI. Régressions multiples entre les paramètres technologiques et les caractères physicochimiques de laits.

\begin{tabular}{|c|c|c|c|c|c|}
\hline & TPR & VRG & $F M G$ & PSE & $D H C$ \\
\hline $\begin{array}{l}\text { Variables } \\
\text { explicatives } \\
\text { (coefficients } \\
\text { de régression } \\
\text { standard) (1) }\end{array}$ & $\begin{array}{l}\text { PST }(-0,635) \\
\text { CAI }(-0,366) \\
\text { MIP }(-0,366) \\
\text { CAT }(-0,271) \\
\text { ASB }(0,263)\end{array}$ & $\begin{array}{l}\text { CNT }(0,675) \\
\text { CNS }(-0,225) \\
\operatorname{BET}(0,215)\end{array}$ & $\begin{array}{l}\text { CNT }(0,551) \\
\text { CAT }(0,325) \\
\text { DHY }(0,301) \\
\text { CNS }(-0,202) \\
\text { DMO }(-0,144)\end{array}$ & $\begin{array}{l}\text { CNT }(-0,459) \\
\text { CAC }(-0,378) \\
\text { CNS }(0,228) \\
\text { AST }(-0,232)\end{array}$ & $\begin{array}{l}\text { PIC }(0,696) \\
\text { CPI }(0,460) \\
\text { ASB }(0,397)\end{array}$ \\
\hline $\begin{array}{l}\text { Coefficients } \\
\text { de corrélation } \\
\text { multiples } \\
\text { associés } \\
\text { aux modèles }\end{array}$ & 0,58 & 0,64 & 0,80 & 0,81 & 0,65 \\
\hline
\end{tabular}

(1) Les coefficients de régression standards attribués, à chaque variable dans le modèle, sont les coefficients de régression multipliés par le rapport écart type de la variable explicative sur écart type de la variable expliquée. Ces coefficients peuvent être comparés entre eux du fait de la pondération apportée par l'écart type qui nivelle les différences d'ordre de grandeur entre les valeurs prises par les différentes variables.

Vitesse de raffermissement et fermeté maximale du gel

Les équations de régression calculées pour ces deux variables technologiques montrent une certaine similitude; plusieurs caractères physicochimiques interviennent en effet dans les deux modèles, ce qui est logique, puisque les deux variables présentent entre elles une corrélation étroite $(r=0,83)$.

Ainsi, la concentration en caséines explique la majeure partie des variations de la vitesse de raffermissement et de la fermeté du gel, comme l'indiquent les coefficients de régression positifs relativement élevés appliqués à cette variable dans les deux équations. Les deux variables technologiques sont également correlées, mais négativement, avec la concentration en caséines non centrifugeables.

Les caractéristiques micellaires ont une incidence sur la fermeté du gel. Celle-ci est correlée positivement avec le degré d'hydratation des micelles, négativement avec leur diamètre moyen. Enfin, la vitesse de raffermissement du gel est correlée positivement avec la proportion de caséine $\beta$, alors que la fermeté de gel est corrélée de manière très significative avec la concentration en calcium total.

\section{Aptitude à l'égouttage}

La corrélation négative relevée entre le poids de sérum égoutté et la concentration en caséines totales ainsi que l'absence de liaison entre cette variable et le degré d'humidité du caillé centrifugé confirment les relations précédemment signalées entre les paramètres de l'aptitude à la coagulation.

Une corrélation positive très significative est observée entre le degré d'humidité du caillé et le rapport caséines $\alpha_{\mathrm{s}}$ sur caséine $\beta$. La corrélation négative relevée 
entre la proportion de caséines $\alpha_{s}$ et le poids de sérum égoutté va dans le même sens. On notera que le degré d'humidité du caillé centrifugé est sensiblement plus élevé dans le cas du lait de vache qui présente un rapport $\alpha_{s} / \beta$ beaucoup plus important que le lait caprin.

Plusieurs variables, liées à la minéralisation des micelles, influent sur l'aptitude des gels à l'égouttage. Ainsi, alors que la concentration en calcium colloïdal a une incidence négative assez marquée sur le poids de sérum égoutté, la teneur en phosphore inorganique colloïdal et le rapport calcium sur phosphore inorganique micellaires sont les deux principaux caractères expliquant les variations du degré d'humidité du caillé centrifugé. Ces corrélations indiquent que l'égouttage du gel présure est réduit par une forte minéralisation des micelles et plus particulièrement une forte minéralisation calcique.

\section{Analyse de corrélation canonique}

Le traitement des résultats par régression multiple a été complété par une analyse de corrélation canonique, méthode statistique globale permettant de mettre en évidence les relations linéaires existant entre deux groupes de variables observées sur un ensemble d'individus (Caillez \& Pages, 1976). Dans le cas présent, les deux groupes sont constitués d'une part des caractères physico-chimiques (groupe 2), et d'autre part des variables technologiques (groupe 1).

Le Tableau VII reproduit l'un des principaux résultats fournis par le programme de calcul : les coefficients de corrélation entre les variables canoniques et les variables initiales. L'analyse de ce tableau et des autres résultats donnés par le programme montre qu'un petit nombre de caractères physico-chimiques explique l'essentiel des variations du comportement au cours de la coagulation des laits caprins individuels. La première variable canonique du groupe 1 (AC1), combinaison linéaire des variables technologiques, est correlée fortement à la fermeté et à la vitesse de raffermissement du gel (positivement) ainsi qu'au poids de sérum égoutté (négativement). II s'agit en quelque sorte d'une variable "synthétique" construite essentiellement à partir de ces 3 variables technologiques. Cette variable canonique est correlée de façon maximale avec la variable $\mathrm{PC} 1$, qui est principalement une combinaison linéaire des variables : concentrations en caséines ( $r=0,849)$, en calcium colloïdal $(r=0,646)$ et en calcium total $(r=0,531)$, diamètre moyen des micelles $(r=-0,580)$, rapport calcium soluble/calcium total $(r=-0,543)$. Ces cinq variables permettent d'expliquer de manière globale l'essentiel des variations du comportement de nos échantillons vis-àvis de la présure, temps de prise exclu. Le coefficient de corrélation entre les deux premières variables canoniques $(R=0,91)$ traduit bien l'étroitesse de la liaison. La concentration en caséines totales est, de loin, le premier facteur responsable des différences d'aptitude à la coagulation entre les laits individuels, notamment en termes de fermeté de gel et de vitesse de raffermissement. Les autres caractères ont une influence qui, bien que significative, est nettement moins marquée. Trois variables caractérisant la fraction calcique, les concentrations en calcium total et en calcium colloïdal et le rapport calcium soluble/calcium total, influent à la fois sur les caractères rhéologiques des gels et sur le temps de prise (Cf AC2 et PC2, Tableau VII). Le diamètre moyen des micelles a une incidence négative sur la fermeté de gel alors que la proportion de caséines $\alpha_{\mathrm{s}}$, ainsi que le rapport $\alpha_{s} / \beta$ influent davantage sur le temps de prise. 
Tableau VII. Analyse de corrélation canonique : coefficients de corrélation entre les variables canoniques et les variables initiales. Groupe $1:$ variables canoniques caractérisant l'aptitude à la coagulation des laits (AC); Groupe 2 : variables canoniques physico-chimiques (PC); $R=$ coefficients de corrélation canoniques.

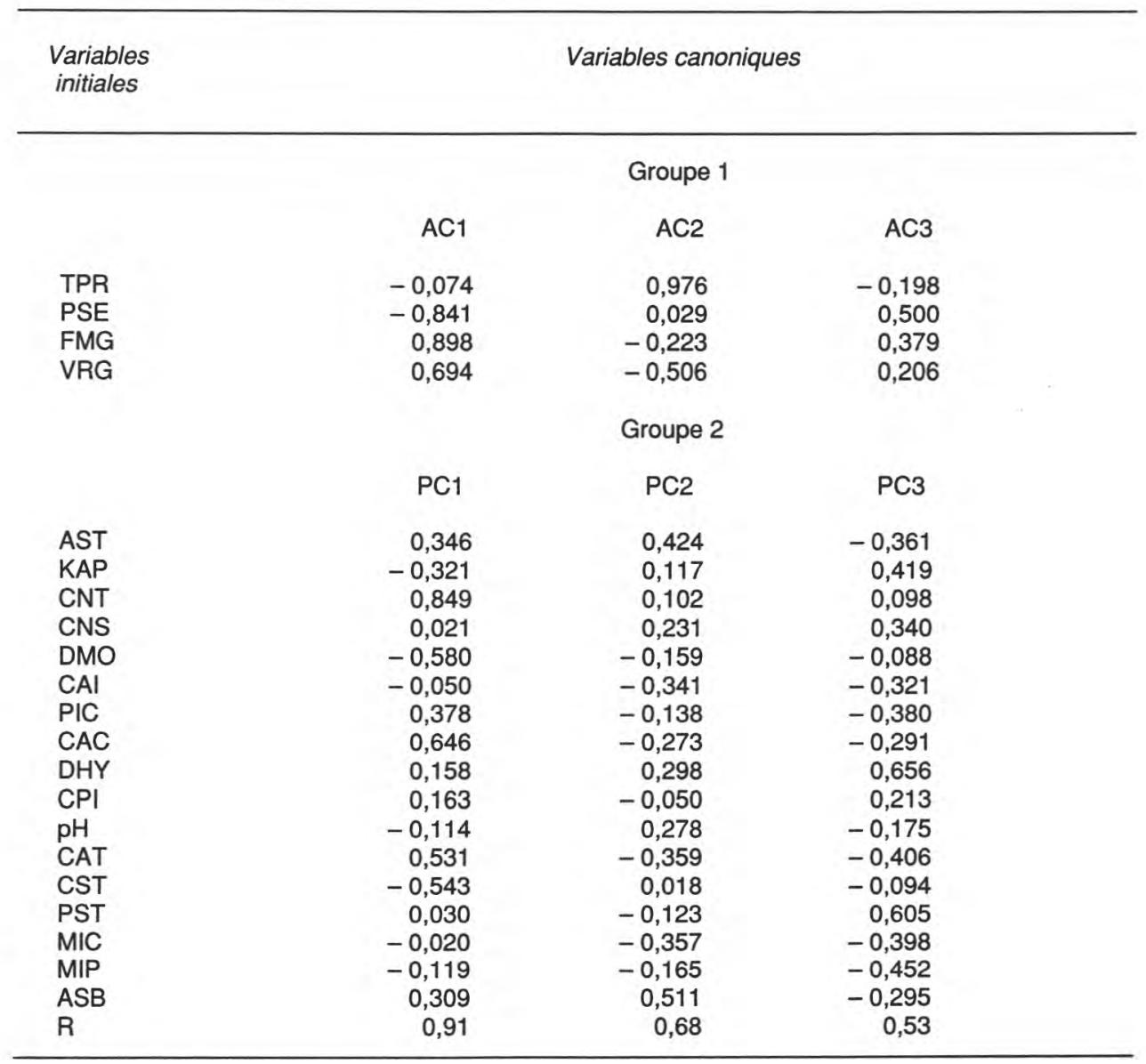

\section{DISCUSSION}

\section{Relations entre caractéristiques}

physico-chimiques

II apparait que, dans le lait de chèvre, les dimensions micellaires sont liées de ma- nière très significative aux proportions relatives de caséines $\alpha_{s}$ et $\beta$ par l'intermédiaire du rapport $\alpha_{s} / \beta$. Dans le lait de vache, il est aussi maintenant bien établi que la composition des micelles détermine leurs dimensions. Ainsi, il est reconnu que la taille des micelles diminue lorsque la proportion de caséine $K$ augmente alors que celle de caséine $\beta$ diminue (e.g. Saito \& Igarashi, 1981; Niki \& Arima, 1984; Don- 
nely et al., 1984). Les relations entre dimensions des micelles et proportion de caséines $\alpha_{s}$ sont en revanche moins clairement établies (Schmidt, 1979; Davies \& Law, 1983; Donnelly et al., 1984; Remeuf et al., données non publiées).

Nos résultats sont en accord avec certains travaux cités précédemment et notamment avec ceux de Schmidt (1979). Tout se passe en effet comme si la substitution des caséines $\alpha_{s}$ par la caséine $\beta$ s'accompagne d'une augmentation de la taille des micelles. La comparaison lait de chèvre/lait de vache vient d'ailleurs appuyer cette observation. En effet, dans le lait bovin, la fraction $\alpha_{\mathrm{s}}$ est la plus importante, et nous avons vu que les micelles ont des dimensions moyennes inférieures aux micelles caprines.

L'existence d'une relation inverse entre la minéralisation des micelles et leur solvatation a été également bien mise en évidence dans le lait de vache (Sood et al., 1979). Cette relation minéralisationsolvatation, confirmée ici sur le lait de chèvre, peut s'expliquer par un double phénomène. Les groupements chargés des acides aminés polaires des caséines constituent des sites de fixation pour les molécules d'eau et les matières salines (Dalgleish \& Parker, 1980). On comprend donc que lorsque la minéralisation des micelles augmente, le nombre de sites de fixation disponibles pour les molécules d'eau devient plus faible, ce qui entraîne une diminution de l'hydratation globale des micelles. Mais parallèlement, c'est aussi la cohésion micellaire qui se trouve renforcée par l'établissement de liaisons protéinesminéraux plus nombreuses. II en résulte que les molécules d'eau ont, physiquement, moins de "place" au sein de la micelle (Snoeren et al., 1984).
Relations entre les variables caracterisant l'aptitude à la coagulation par la présure des laits

Les corrélations significatives mises en évidence entre variables décrivant le comportement des laits vis-à-vis de la présure permettent de mieux "éclairer» les différences observées entre lait de chèvre et lait de vache en ce qui concerne les paramètres de la coagulation. Ainsi, la corrélation négative observée entre le temps de prise et la vitesse de raffermissement du gel concorde avec l'observation selon laquelle le lait de vache présente un temps de coagulation plus long que le lait de chèvre et une vitesse de raffermissement plus faible. En revanche, une question se pose : pourquoi le lait de chèvre, qui présente une vitesse de raffermissement élevée, ne donne-t-il pas un gel de fermeté plus grande que le lait de vache, puisque la fermeté et la vitesse de raffermissement du gel sont étroitement correlées ? Une explication à cette contradiction apparente peut être avancée en considérant certaines équations de modélisation qui décrivent l'évolution de la fermeté du gel en fonction du temps selon une cinétique du $1^{\mathrm{er}}$ ordre (e.g. Douillard, 1973; Gervais \& Cerf, 1983; Douillard, 1986). Dans ce type de modèle, la vitesse de raffermissement initiale est proportionnelle à la fermeté maximale du gel par l'intermédiaire d'une constante de vitesse $\mathrm{k}$. On peut penser que, du fait de l'instabilité particulière des micelles caprines liée à leurs caractéristiques physico-chimiques propres, $k$ prend des valeurs plus élevées dans le lait de chèvre que dans le lait de vache, et l'on aurait alors des vitesses de raffermissement du gel caprin supérieures, malgré des valeurs de fermeté maximale nettement plus faibles. 
Relations entre les caractères physicochimiques des laits et leur comportement vis-à-vis de la présure

Une corrélation négative entre le temps de coagulation et la concentration en calcium total du lait a été observée, sur lait de vache, par de nombreux auteurs (e.g. Mocquot et al., 1954; Auriol, 1961; Ramet \& Weber, 1980; Storry et al., 1983; Tervala \& Antila, 1985). Cette corrélation est donc également vérifiée dans le lait de chèvre. D'autres auteurs ont relevé l'incidence du phosphore inorganique dans le processus de coagulation. Storry et al. (1983) trouvent une corrélation négative entre le temps de coagulation et la teneur du lait en phosphore inorganique total. Mac Mahon et al. (1984) observent que l'addition au lait de phosphate de potassium à des concentrations allant jusqu'à $10 \mathrm{mM}$ entraîne une diminution du temps de coagulation. II semble que la teneur en phosphate de calcium colloïdal soit un facteur primordial de l'aptitude du lait à la coagulation par la présure (Pyne \& Mac Gann, 1962; Zittle, 1970; Shalabi \& Fox, 1982). Nos observations, qui montrent que le temps de prise diminue lorsque le rapport phosphore inorganique colloïdal sur caséine augmente, vont bien dans ce sens.

La corrélation positive mise en évidence entre le temps de prise et le rapport caséines $\alpha_{s}$ sur caséine $\beta$ confirme les résultats obtenus par différents auteurs. Ainsi, Ciafarone \& Addeo (1984) relèvent que les laits de chèvre pauvres en caséine $\alpha_{s 1}$, donc en caséines $\alpha_{s}$ totales, ont un temps de coagulation inférieur à celui des autres. Pearse et al. (1986) observent, sur lait de vache, qu'une augmentation de la proportion de caséine $\beta$ dans les micelles - donc une diminution du rapport $\alpha_{s} / \beta-$ s'accompagne d'un raccourcissement du temps de coagulation.
L'influence de la concentration en caséines sur les caractères rhéologiques du gel présure (vitesse de raffermissement et fermeté du gel) a été établie, sur lait de vache, dans plusieurs études (e.g. Alais, 1965; Dalgleish, 1980; Storry \& Ford, 1982; Storry et al., 1983; Banks \& Muir, 1984). Selon Mac Mahon \& Brown (1984), lorsque la concentration en caséines augmente, le volume de la phase aqueuse et la distance moyenne entre les micelles diminuent. La fréquence des collisions entre micelles de paracaséine est alors plus élevée et il en résulte une augmentation de la vitesse d'agrégation. Le gel aurait ainsi tendance à se former et à se raffermir plus rapidement. La densité du réseau étant plus grande, la consistance du coagulum serait également plus élevée.

L'influence de la concentration en caséines non centrifugeables sur la fermeté du gel présure n'a pas encore, à notre connaissance, été signalée sur lait de chèvre. Dans le lait de vache, différents auteurs ont observé que, sous l'effet d'un refroidissement, la concentration en caséines solubles augmente et parallèlement, le gel présure est moins ferme (Ali et al., 1980; Ichilczyk-Leone et al., 1981).

Les travaux relatifs à l'incidence des dimensions micellaires sur le comportement du lait vis-à-vis de la présure concernent le lait de vache et la plupart ont porté sur la relation taille des micelles/temps de coagulation (Kosikowski \& Mocquot, 1958; Sabarwal \& Ganguli, 1973; Ekstrand et al., 1981). Niki \& Arima (1984) ont cependant mis en évidence des résultats concordants avec les nôtres. Par reconstitution de laits contenant des micelles de tailles différentes, séparées par ultracentrifugation, ils observent que les laits contenant des petites micelles présentent une vitesse de raffermissement et une fermeté de gel plus élevées que ceux qui contiennent de grosses micelles. D'autres études ont 
abouti à des conclusions similaires (Waagner Nielsen et al., 1982; Chahed, 1985) et récemment, dans le cadre d'une recherche visant à préciser les relations entre les caractères physico-chimiques des laits de vache individuels et leur comportement fromager, nous avons pu confirmer l'incidence négative du diamètre moyen des micelles sur la fermeté du gel (Remeuf et al., données non publiées). D'après des observations effectuées en microscopie électronique, il semble que les petites micelles fusionnent plus étroitement entre elles que les particules de taille élevée. II en résulterait la formation d'un gel-présure plus cohérent et plus ferme (Niki \& Arima, 1984). On relèvera enfin que la comparaison lait de chèvre/lait de vache est en accord avec ces observations, puisque le lait caprin présente des micelles en moyenne plus grosses que le lait bovin et un gel-présure moins ferme.

La corrélation positive entre vitesse de raffermissement du gel et proportion de caséine $\beta$ est en contradiction apparente avec les relations précédemment relevées entre dimensions des micelles et vitesse de raffermissement; elle rejoint cependant les observations faites par d'autres auteurs sur lait de vache (Yun et al., $1982 \mathrm{a}$ et $b$; Pearse et al., 1986) selon lesquelles les groupements phosphorylés de la caséine $\beta$, localisés dans la partie $\mathrm{N}$ terminale de la chaîne, joueraient un rôle privilégié dans les interactions entre micelles lors du processus de coagulation. Toutefois, Pearse et al. (1986) notent une influence de la proportion de caséine $\beta$ sur la fermeté de gel mais non sur la vitesse de raffermissement.

L'influence de la concentration en calcium total sur la fermeté du gel a été bien établie dans le cas du lait de vache (Mocquot et al., 1954; Auriol, 1961; Pyne, 1962; Storry et al., 1983; Tervala \& Antila, 1985) et nos résultats confirment sur lait de chèvre l'importance de ce paramètre.
Moins connue est l'incidence négative d'une forte minéralisation des micelles sur l'aptitude du gel présure à l'égouttage pour laquelle nos observations rejoignent celles faites sur lait de vache par d'autres auteurs (e.g. Lombard, 1982; Storry et al., 1983). II est possible que le phosphate de calcium colloïdal exerce cette influence négative par un effet d'encombrement de sites actifs, entraînant une diminution des interactions micellaires au cours de la synérèse; le gel aurait alors une capacité de contraction et par suite d'exsudation de lactosérum moindre.

L'ensemble de ces données établies sur lait de chèvre apporte aussi des éléments de réponse au problème soulevé par les différences d'aptitude à la coagulation par la présure entre lait de chèvre et lait de vache.

Disposant, avec l'étude conduite parallèlement sur lait de vache (Remeuf et al., données non publiées), de résultats comparables sur une quarantaine d'échantillons individuels, il a été possible, par sélection d'échantillons de lait de chèvre à teneur en caséine élevée et d'échantillons de laits de vache à teneur en caséine faible, de constituer deux groupes de laits présentant des taux de caséines proches. Les caractéristiques physico-chimiques et technologiques moyennes de ces laits sont indiquées respectivement dans les $\mathrm{Ta}$ bleaux VIII et IX. Se trouve ainsi bien confirmée la différence importante d'aptitude à la coagulation par la présure entre les laits des deux espèces, même à teneurs en caséines égales, qui a été déjà signalée par Storry et al. (1983).

Les équations de régression calculées précédemment ont été appliquées au lait de vache "moyen" dont les caractéristiques de composition sont les valeurs moyennes des 30 échantillons sélectionnés. En d'autres termes, la valeur de cha- 
Tableau VIII. Caractéristiques physico-chimiques moyennes de laits de chèvre et de vache individuels, sélectionnés sur la base d'une teneur en caséines identique.

\begin{tabular}{lrr}
\hline & Lait de chèvre & Lait de vache \\
\hline Concentration en caséines & 24,45 & 24,45 \\
totales (g/l) & 1,96 & 0,76 \\
Concentration en caséines & & \\
solubles (g/l) & 28,0 & 59,8 \\
AST & 53,7 & 31,2 \\
BET & 18,3 & 8,9 \\
KAP & 0,617 & 1,91 \\
ASB & 245 & 177 \\
DMO & 1,77 & 2,06 \\
DHY & 1,26 & 1,08 \\
CAT & 35,3 & 30,3 \\
MIC & 14,68 & 10,30 \\
MIP & & \\
\hline
\end{tabular}

que paramètre technologique a été estimée à partir des modèles de régression, en attribuant aux variables explicatives les valeurs moyennes calculées pour les 30 échantillons sélectionnés. Disposant, pour chaque paramètre technologique, des valeurs réelles et des valeurs estimées, il est possible de vérifier dans quelle mesure les caractères physico-chimiques qui expliquent les variations de l'aptitude à la coagulation des laits de chèvre, permettent également d'interpréter les différences de comportement entre lait de chèvre et lait de vache (Tableau IX).

Tableau IX. Essai d'interprétation des différences d'aptitude à la coagulation par la présure entre laits de chèvre et laits de vache à partir des équations de régression obtenues sur lait de chèvre.

\begin{tabular}{|c|c|c|c|c|c|c|}
\hline \multirow{2}{*}{$\begin{array}{l}\text { Caractères } \\
\text { technologiques }\end{array}$} & \multicolumn{2}{|c|}{$\begin{array}{l}\text { Lait de chèvre } \\
\text { (Groupe } X, n=37 \text { ) }\end{array}$} & \multicolumn{2}{|c|}{$\begin{array}{c}\text { Lait de vache } \\
\text { (Groupe } Y, n=30 \text { ) }\end{array}$} & \multirow[b]{2}{*}{$e^{*}$} & \multirow[b]{2}{*}{$f^{\star}$} \\
\hline & $\begin{array}{c}\text { mesuré } \\
a\end{array}$ & $\int_{b}^{C a l c u l e ́ ~}$ & $\begin{array}{c}\text { mesuré } \\
c\end{array}$ & $\begin{array}{c}\text { calculé } \\
d\end{array}$ & & \\
\hline FMG & 63,4 & 60,4 & 85,5 & 81,3 & $94,6 \%$ & DHY/DMO \\
\hline VRG & 5,06 & 4,94 & 2,38 & 4,25 & $25,7 \%$ & BET \\
\hline TPR & 19,18 & 19,91 & 37,53 & 29,95 & $54,7 \%$ & ASB \\
\hline PSE & 18,37 & 18,37 & 17,99 & 18,05 & $85,8 \%$ & AST \\
\hline $\mathrm{DHC}$ & 1,69 & 1,70 & 2,36 & 2,15 & $67,6 \%$ & ASB \\
\hline
\end{tabular}

(b, d) : calculés en appliquant les équations de régression obtenues sur lait de chèvre aux valeurs moyennes des variables explicatives correspondant aux deux laits; (e) : écart "expliqué" = rapport écart calculé/écart réel mesuré $=(\mathrm{d}-\mathrm{b}) \times 100 /(\mathrm{c}-\mathrm{a}) ;(\mathrm{f})$ : variables prépondérantes pour expliquer l'écart calculé. 
II apparaît ainsi que la différence de fermeté de gel observée entre laits bovin et caprin, indépendamment des concentrations en caséines, est très bien expliquée par les différences de caractères physicochimiques entre les deux laits, puisque l'écart entre les valeurs de fermeté calculées représente $94,6 \%$ de l'écart réel mesuré. Deux variables ont une influence déterminante pour expliquer cet écart : le diamètre des micelles et leur degré d'hydratation.

L'écart de temps de prise entre les deux laits n'est expliqué qu'en partie $(54,7 \%)$ par les différences de caractéristiques physico-chimiques prises en compte. Le rapport caséines $\alpha_{s}$ sur caséine $\beta$ joue ici un rôle prépondérant et le fait que la différence de temps de coagulation entre lait de chèvre et lait de vache pourrait être liée, au moins en partie, aux différences des proportions relatives de caséines $\alpha_{\mathrm{s}}$ et $\beta$ se trouve ainsi confirmé.

Une conclusion similaire peut être avancée en ce qui concerne l'aptitude à l'égouttage puisque la proportion de caséines $\alpha_{s}$ totales ou le rapport $\alpha_{s} / \beta$ expliquent une grande partie des écarts existant entre les deux laits en ce qui concerne le poids de sérum égoutté et le degré d'humidité du caillé centrifugé. Pour la vitesse de raffermissement du gel, les résultats sont moins concluants, le modèle de régression ne permettant d'expliquer que $25,7 \%$ de l'écart observé. Outre la proportion de caséine $\beta$, il existe probablement d'autres facteurs explicatifs non pris en compte dans ce travail.

\section{CONCLUSION}

Cette étude complète sur différents points les données de la littérature, relatives aux caractéristiques physico-chimiques et au comportement technologique du lait de chèvre. Elle contribue ainsi à une meilleure connaissance de ce lait en tant que matière première de fromagerie.

La variabilité des caractéristiques physico-chimiques et technologiques des laits individuels a permis de mettre en évidence un certain nombre de corrélations significatives entre l'aptitude des laits à la coagulation par la présure et leur composition protéique et minérale. Les caractères physico-chimiques ayant une influence prépondérante sont les concentrations en caséines totales, en calcium total et en calcium colloïdal, le rapport calcium soluble sur calcium total, le diamètre moyen des micelles, le rapport caséines $\alpha_{s}$ sur caséine $\beta$.

Les données acquises ont également permis de préciser les causes du comportement fromager particulier du lait de chèvre. Ainsi, en dehors de la concentration en caséines, l'écart de fermeté entre les coagulums bovin et caprin est essentiellement dû aux différences de caractéristiques micellaires (diamètre moyen et degré d'hydratation des micelles) des deux laits, ces caractéristiques étant d'ailleurs elles mêmes liées à la composition des micelles. Les corrélations obtenues par régression multiple montrent en effet que lorsque la minéralisation des micelles augmente, leur degré d'hydratation diminue. Quant au diamètre moyen, il est essentiellement lié au rapport caséines $\alpha_{s}$ sur caséine $\beta$ : il a tendance à diminuer lorsque ce rapport augmente. Les proportions relatives de caséines $\alpha_{s}$ et $\beta$ permettent aussi d'expliquer en partie les différences entre les laits bovin et caprin concernant le temps de prise, la vitesse de raffermissement du gel et l'aptitude à l'égouttage.

Sans doute certains résultats peuventils, a priori, surprendre ou faire apparaître des contradictions difficiles à cerner. On 
relèvera par exemple qu'il n'a pas été observé de relation entre le $\mathrm{pH}$ originel des laits et leur aptitude à la coagulation, alors qu'il s'agit pourtant d'un paramètre déterminant. II est possible que les variations de $\mathrm{pH}$ soient dans le cas présent d'amplitude trop faible pour être réellement influentes ou qu'elles s'accompagnent de modifications d'autres caractéristiques physico-chimiques qui masquent leur effet propre. Certaines corrélations ne sont pas non plus sans ambiguïté; il en est ainsi de celles concernant la vitesse de raffermissement du gel, la proportion de caséine $\beta$ et les dimensions des micelles, ou, dans la comparaison lait de vache/lait de chèvre, les corrélations entre raffermissement du gel et fermeté maximale qui sont étroites dans l'un et l'autre cas et qui cependant évoluent en sens opposé lorsqu'on confronte les deux laits.

L'ensemble de ces données n'en fournit pas moins des indications intéressantes pour la recherche de moyens permettant d'améliorer le comportement fromager des laits de chèvre. Ainsi, dans le cadre de l'application de traitements de correction technologique au lait, ce sont ceux visant à augmenter la teneur en caséines ou à modifier les équilibres calciques qui devraient avoir le plus d'impact. Ces traitements sont aussi ceux qui, industriellement, sont les plus aisément applicables. En effet, il paraît difficile, dans l'état actuel de la technique, d'imaginer des procédés permettant de modifier les dimensions des micelles ou les proportions relatives de caséines. Par ailleurs, il reste à montrer, par une expérimentation à plus grande échelle, sur une population de chèvres représentative du cheptel caprin français, qu'une sélection des animaux sur la base de critères tels les proportions de caséines $\alpha_{s}$ ou les dimensions micellaires, permettrait une amélioration effective de la qualité fromagère des laits, sans répercussions dommageables sur d'autres caractères importants, notamment la quantité de matière utile produite par lactation, la rusticité ou la fécondité des animaux. A ce stade le polymorphisme génétique de la caséine $\alpha_{s 1}$, dont on sait qu'il détermine les proportions des caséines $\alpha_{s}$ doit naturellement être pris en compte. Les travaux du laboratoire de technologie de I'INA ParisGrignon concernant l'incidence de ce polymorphisme sur les aptitudes fromagères des laits feront l'objet d'une prochaine publication.

\section{REMERCIEMENTS}

Ce travail a été réalisé dans le cadre d'une convention entre I'INRA et la région PoitouCharentes, relative à un programme de recherche sur la technologie du lait de chèvre. II a permis des contacts fructueux avec les instances régionales à qui nous adressons nos remerciements.

Nos remerciements vont également à $M$. Sauvant, directeur de la station de nutrition et alimentation INRA de I'INA-PG, pour ses conseils et l'intérêt qu'il a manifesté pour ce travail, et à M. Hervieu, responsable du troupeau caprin, pour son aide et sa disponibilité lors des prélèvements d'échantillons.

\section{RÉFÉRENCES}

Abou-Dawood A.E. \& El-Sawaf S. (1977) Curd tension of milk from some mammals in comparison to some infant milk powder. Egypt. J. Dairy Sci. $5,129-133$

Alais C. (1965) Influence de divers traitements sur l'aptitude du lait à la coagulation par la présure. Rev. Lait. Fr. 218, 90-93

Ali E.L., Andrews A.T. \& Cheeseman G.C. (1980) Influence of storage of milk on casein distribution between the micellar and soluble 
phases and its relationship to cheese-making parameters. J. Dairy Res. 47, 371-382

Assenat L. (1967) Contribution à l'étude d'une méthode d'identification des laits et fromages au moyen de l'électrophorèse en gel de polyacrylamide. Lait 47, 393-414

Auriol P. (1961) Quelques facteurs de variation du temps de coagulation des laits individuels de vache. Ann. Biol. Anim. Biochim. Biophys. 1, 152-162

Banks J.M. \& Muir D.D. (1984) Coagulum strength and cheese yield. Dairy Ind. Int. 49, 1721,36

Blattner C. \& Gallmann P. (1980) Vergleichende untersuchungen zur labgerinnung von Ziegen und Kulhmilch. Schweiz. Milchzeitung 106, 277 278

Boulanger A. (1976) Etude biochimique et génétique des protéines du lait de chèvre (Capra hircus). Thèse $3^{\theta}$ cycle. Université Paris VII

Burnett J. \& Scott Blair G.W. (1963) A speedcompensated torsiometer for measuring the setting of milk by rennet. Dairy Ind. 28, 220-223

Caillez F. \& Pages J.P. (1976) Introduction à l'analyse des données. SMASH, Paris

Chahed S. (1985) Rapports entre les dimensions des micelles de caséine, leur composition et leur comportement vis-à-vis de la présure. Mémoire DEA science alimentaire lait et produits laitiers, université de Caen-INA PG.

Ciafarone N. \& Addeo F. (1984) Composition de la caséine et propriétés du lait de chèvre. Vergaro (11), 17-24

Dalgleish D.G. (1980) Effect of milk concentration on the rennet coagulation time. J. Dairy Res. 47, 231-235

Dalgleish D.G. \& Parker T.G. (1980) Binding of calcium ions to bovine $\alpha_{\mathrm{s} 1}$-casein and precipitability of the protein-calcium ion complexes. $J$. Dairy Res. 47, 113-122

Davies D.T. \& Law A.J.R. (1983) Variation in the protein composition of bovine casein micelles and serum casein in relation to micellar size and milk temperature. J. Dairy Res. 50, 67-75

Donnelly W.J., Mc Neill G.P., Buchheim W. \& Gann T.C.A. (1984) A comprehensive study of the relationship between size and protein composition in natural bovine casein micelles. Biochim. Biophys. Acta 789, 136-143

Douillard R. (1973) Rheological analysis of curd formation. J. Texture Stud. 4, 158-165
Douillard R. (1986) Modèle cinétique de la coagulation et de la gélification du lait sous l'action de protéases. Lebensm. Wiss. Technol. 19, 202-207

Ekstrand B., Larsson-Raznikiewicz M., Brannang E. \& Swenson C. (1981) Size distribution of casein micelles related to coagulation properties. Swed. J. Agric. Res. 11, 57-61

Gervais A. (1979) Contribution à l'étude de la mesure de la rigidité des laits gélifiés par la présure. DEA sciences et techniques de l'industrie alimentaire, ENSIA Massy

Gervais A. \& Cerf O. (1983) Comparison of two models for fitting rennet-induced milk coagulation kinetics. J. Texture Stud. 14, 47-59

Grosclaude F., Mahe M.F., Brignon G., Di Stasio L. \& Jeunet R. (1987) A mendelian polymorphism underlying quantitative variations of goat $\alpha_{s 1}$ casein. Génét. Sél. Evol. 19, 399-412

Ichilczyc-Leone J., Amram Y., Schneid N. \& Lenoir J. (1981) Le refroidissement du lait et son comportement en fromagerie. Rev. Lait. Fr. 401, 7-14

Jenness R. (1980) Composition and characteristics of goat milk : a Review 1968-1979. J. Dairy Sci. 63, 1605-1630

Kalatzopoulos G. (1970) Contribution to the study of some rheological properties of cheese curd. Thèse, Collège d'Agriculture d'Athènes, Grèce

Kosikowski F.V., Mocquot G. (1958) Progrès de la technologie du fromage. FAO Rome

Le Mens P. (1983) Le lait de chèvre (sa composition biochimique et les problèmes posés à l'industrie formagère). Mémoire CNAM, Paris

Lin S.H.C., Dewan R.K., Bloomfield V.A. \& Morr C.V. (1971) Inelastic light-scattering study of the size distribution of bovine milk casein micelles. Biochemistry 10, 4788-4793

Lombard S.H. (1982) Factors affecting enzymatic coagulation of milk and syneresis. $\mathrm{In}$ : Miles Annual Cheesemakers symposium. Dep. of Food Science, University of Pretoria, South Africa

Mac Mahon D.J. \& Brown R.J. (1984) Enzymic coagulation of casein micelles : a review. $J$. Dairy Sci. 67, 919-929

Mac Mahon D.J., Brown R.J., Richardson G.H. \& Ernstrom C.A. (1984) Effects of calcium, phosphate, and bulk culture media on milk coagulation properties. J. Dairy Sci. 67, 930-938 
Mocquot G., Alais C. \& Chevalier R. (1954) Etude sur les défauts de coagulation du lait par la présure. Ann. Technol. Agric. 3, 1-44

Niki R. \& Arima S. (1984) Effects of size of casein micelle on firmness of rennet curd. Jpn. J. Zootech. Sci. 55, 409-415

Nilsen K.O. \& Abrahamsen R.K. (1985) Difficulties in measuring the syneresis of goat milk rennet curd by dilution of an added tracer. J. Dairy Res. 52, 209-212

O'Connor P. \& Fox P.F. (1977) The proteins and salts of some nonbovine milks. J. Dairy Res. 44, 607-609

Parkash S. \& Jenness R. (1968) The composition and characteristics of goat's milk : a review. Dairy Sci. Abstr. 30, 67-87

Pearse M.J., Linklater P.M., Hall R.J. \& Mackinlay A.G. (1986) Effect of casein micelle composition and casein dephosphorylation on coagulation and syneresis. J. Dairy Res. 53, 381-390

Pyne G.T. (1962) Reviews of the progress of Dairy Science : some aspects of the physical chemistry of the salts of milk. J. Dairy Res. 29, 101-130

Pyne G.T. \& Mac Gann C.A. (1962) The influence of the colloidal phosphate of milk on the rennet coagulation. In : XVI International Dairy Congress. vol B, section IV, 611-616

Ramet J.P. \& Weber F. (1980) Contribution à l'étude de l'influence des facteurs de milieu sur la coagulation enzymatique du lait reconstitué. Lait 60, 1-13

Ramos M. \& Juarez (1981) The composition of ewe's and goat's milk. Int. Dairy Fed. Bull. 140

Remeuf F. \& Lenoir J. (1985) Caractéristiques physico-chimiques de laits de chèvre et leur aptitude à la coagulation par la présure. Rev. Lait. Fr. (446), 32-34, 37-38, 40

Richardson B.C., Creamer L.K., Pearce K.N. \& Munford R.E. (1974) Comparative micelle structure. II. Structure and composition of casein micelles in ovine and caprine milk as compared with those in bovine milk. J. Dairy Res. 41, 239247

Sabarwal P.K. \& Ganguli N.C. (1973) Rennet susceptibility of ultracentrifugal milk serum fractions as revealed by turbidity measurement. Indian J. Dairy Sci. 26, 140-142

Saito Z. \& Igarashi Y. (1981) Compositional properties and voluminosity of centrifugaliy fractionated casein micelles of cow's milk. Bull. Fac. Agric. Hirosaki Univ. 35, 1-11
Schmidt D.G. (1979) Properties of artificial casein micelles. J. Dairy Res. 46, 351-355

Shalabi S.I. \& Fox P.F. (1982) Influence of pH on the rennet coagulation of milk. $J$. Dairy Res. 49, 153-157

Snoeren T.H.M., Klok H.J., Van Hooydonk A.C.M. \& Danman A.J. (1984) The voluminosity of casein micelles. Milchwissenschaft 39, 461463

Sood S.M., Gaind D.K. \& Dewan R.K. (1979) Correlation between micelle solvation and calcium content. N. Z. J. Dairy Sci. Technol. 14, 32-34

Steinsholt K. (1973) The use of an Instron Universal Testing Instrument in studying the rigidity of milk during coagulation by rennin. Milchwissenschaft 28, 94-97

Storry J.E. \& Ford G.D. (1982) Some factors affecting the post clotting development of coagulum strength in renneted milk. J. Dairy Res. 49, 469-477

Storry J.E., Grandison A.S., Millard D., Owen A.J. \& Ford G.D. (1983) Chemical composition and coagulating properties of renneted milks from different breeds and species of ruminant. J. Dairy Res. 50, 215-229

Tervala H.L. \& Antila V. (1985) Factors affecting the renneting properties of milk. Meijeritiet. Aikak. 43, 16-25

Thompson M.P., Boswell R.T., Martin V., Jenness R. \& Kiddy C.A. (1969) Casein-pellet solvation and heat stability of individual cow's milk. J. Dairy Sci. 52, 796-798

Uriel J. (1966) Méthode d'électrophorèse dans les gels d'acrylamide-agarose. Bull. Soc. Chim. Biol. 48, 969-981

Wagner Nielsen E., Berntsen G., Hansen S., Larson B.V. \& Edelsten D. (1982) Rennet coagulation of casein micelles of different size. In : XXI International Dairy Congress (brief communications), Vol. 1, Book 2, 257-258. Mir Moscou

Yun S.E., Ohmiya K., Shimizu S. (1982 a) Role of B-casein in milk curdling. Agric. Biol. Chem. 46, 443-449

Yun S.E., Ohmiya K. \& Shimizu S. (1982 b) Role of the Phosphoryl Group of B-casein in milk curdling. Agric. Biol. Chem. 46, 1 505-1 511

Zittle C.A. (1970) Influence of phosphate and other factors on the rennin gel obtained with whole casein and with K-casein in the presence of calcium salts. J. Dairy Sci. 53, 1, 013-1 017 